\title{
IMPROVED BLOCK BASED FEATURE LEVEL IMAGE FUSION TECHNIQUE USING CONTOURLET WITH NEURAL NETWORK
}

\author{
C.M.Sheela Rani ${ }^{1}$, P.S.V.Srinivasa Rao $^{2}$ and V.VijayaKumar ${ }^{3}$ \\ ${ }^{1}$ Department of Computer Science and Engineering, Research scholar, Acharya \\ Nagarjuna University, Guntur, Andhra Pradesh, India \\ sheelarani11@yahoo.com \\ ${ }^{2}$ Department of Computer Science and Engineering, Principal, Rao \& Naidu Engg. \\ College, Ongole, Andhra Pradesh, India \\ parimiraodyahoo.com \\ ${ }^{3}$ Department of Computers, Head SRRF, GIET, JNTUK, \\ Rajahmundry, Andhra Pradesh, India \\ vijayvakula@yahoo.com
}

\begin{abstract}
As multisensory data is made available in many areas such as remote sensing, medical imaging, etc, the sensor fusion has become a new field for research. Multisensor image fusion mainly focuses on combining spatial information of a high resolution panchromatic (PAN) image with spectral information of a low resolution multispectral image (MS) to produce an image with highest spatial content while preserving spectral resolution. A geometrical transform called contourlet transform (CT) is introduced, which represents images containing contours and textures. This paper derived an efficient block based feature level contourlet transform with neural network (BFCN) model for image fusion. The proposed BFCN model integrates $C T$ with neural network $(N N)$, which plays a significant role in feature extraction and detection in machine learning applications. In the proposed BFCN model, the two fusion techniques, CT and NN are discussed for fusing the IRS-1D images using LISS III scanner about the locations Hyderabad, Vishakhapatnam, Mahaboobnagar and Patancheru in Andhra Pradesh, India. Also Landsat 7 image data and QuickBird image data are used to perform experiments on the proposed BFCN model. The features under study are contrast visibility, spatial frequency, energy of gradient, variance and edge information. Feed forward back propagation neural network is trained and tested for classification, since the learning capability of NN makes it feasible to customize the image fusion process. The trained NN is then used to fuse the pair of source images. The proposed BFCN model is compared with other techniques to assess the quality of the fused image. Experimental results clearly prove that the proposed BFCN model is an efficient and feasible algorithm for image fusion.
\end{abstract}

\section{KEY WORDS}

Image fusion, Contourlet Transform, Neural Network, block based features, performance measures.

\section{INTRODUCTION}

In remote sensing, multispectral scanners are used to acquire the images over multiple ranges of spectrum. As these sensors are of low spatial resolution i.e., less number of pixels, all the small details are hidden. The panchromatic sensors are of high spatial resolution but rendered in black and white. With a fusion process, an unique image can be achieved containing the spatial resolution of PAN image and the spectral resolution of MS image. Particularly, 
Signal \& Image Processing : An International Journal (SIPIJ) Vol.3, No.4, August 2012

multisensor image fusion is the process of combining relevant information from the registered images taken from the same scene in order to achieve a high quality fused image, which contains the best information coming from the source images. Multisensor data fusion combines information from the multiple sensors and sources to integrate the information that are not possible using a single sensor. Few requirements imposed on the fusion result are - (a) the fused image should contain all relevant information contained in both the images (b) the fusion process should not introduce any artefacts or inconsistencies (c) irrelevant features and noise should be totally suppressed.

Many researchers worked on pixel level image fusion. Thus excess of pixel level fusion algorithms have been developed [1], [2] with different performance and complexity characteristics. Siddiqui et al. proposed an algorithm for block-based feature-level multi-focus image fusion in [3]. G. Piella proposed a region-based multi-resolution image fusion algorithm which combines the aspects of region and pixel-based fusion [4]. A. Toet proposed an algorithm for image fusion by a ratio of low pass pyramid [5]. K. Kannan et al. evaluated the performance of all the levels of multi-focused images using different wavelet transforms [6]. Dong et al. discussed various advances in multi-sensor data fusion [7]. Riazifar et al. proposed a compression scheme in transform domain and compared the performance of both DWT and CT [8]. Eslami et al. proposed a new image coding scheme based on the wavelet-based contourlet transform using a contourlet-based set partitioning in hierarchical trees algorithm. Eslami et al. proposed hybrid wavelets and DFB (HWD) transform for improvement over the wavelet and contourlet transforms [9]. Eslami et al. also proposed a wavelet-based contourlet transform (WBCT) which is capable of approximating natural images containing contours and oscillatory patterns [10] but the major disadvantage is the numbers of directions are doubled at every other wavelet level. Khosravi. et al. proposed a block feature based image fusion algorithm which integrates multiwavelet transforms with the feed forward probabilistic neural networks for the images which are in and out of focus [11]. Nava et al. presented a technique to construct a multiscale representation of planar contours based on the multiwavelet transform. Starck et al. described the digital implementation of the ridgelet transform and the curvelet transform [12].

The section 2 deals with image fusion based on contourlet transform; section 3 describes about artificial neural networks and the proposed algorithm; section 4 deals with quality assessment techniques; section 5 deals with results and discussions; conclusions in section 6 .

\section{IMAGE FUSION BASED ON CONTOURLET TRANSFORM}

The discrete wavelet transform (DWT) is the commonly used transform for image fusion at multiscale, since it minimizes the structural distortions. DWT is a very useful tool to represent images containing smooth areas separated with edges but cannot perform well when the edges are smooth curves. The DWT cannot capture the image geometry structure. The major drawback for wavelets in 2-D is their limited ability in capturing directional information. The 2-D DWT ignores the discontinuities at horizontal and vertical edges [13]. In a 2-D DWT, a signal passes through low pass and high pass analysis filter banks followed by a decimation operation, along Xdimension and Y- dimension separately. In [14], the main disadvantages of wavelet transforms mentioned are : directionality - the basis elements defined in a variety of directions and anisotropy - the basis elements defined in various aspect ratios and shapes.

To overcome the above deficiencies of the wavelet transforms, recently some researchers have proposed few multiscale and directional representations that can capture the intrinsic geometrical structures such as smooth contours existing in the images. Some examples include complex wavelets [15], curvelet [16] and contourlet [17]. After much research in directional transforms, a new geometrical transform called contourlet transform (CT) is introduced, which represents images containing contours and textures. A directional extension of multidimensional 
Signal \& Image Processing : An International Journal (SIPIJ) Vol.3, No.4, August 2012

wavelet transform is a CT that aims to capture curves instead of points, and provides for directionality and anisotropy. The CT was introduced by Do and Vetterli [18]. It has the property of capturing contours and fine details in the images. It is computationally efficient, as it defines an approximation property for smooth 2D functions and finds a direct discrete-space construction. It has the advantages of multiscale localization, directionality and anisotropy. It is a multiresolution transform which uses laplacian pyramid (LP) and a directional filter bank (DFB). The LP decomposes images into subbands and DFB analyzes each detail image. Hence, contourlet transform is a double filter bank structure.

In 2002, Do M N and Vetterli M proposed that CT represents images using basis elements having a variety of elongated shapes with different aspect ratios.This transform is suitable for applications involving edge detection with high curve content. In 2009, [19] proposed an algorithm for multi-focus image fusion using wavelet based contourlet transform and region.

\section{Artificial Neural Networks}

The Artificial Neural Network (ANN) based method employs a nonlinear response function that iterates many times in a special network structure in order to learn the complex functional relationship between input and output training data. For any neural network, there will be three layers - input layer, hidden layer and output layer. The input layer has several neurons, which represent the feature factors extracted and normalized from the source images. The hidden layer has several neurons and the output layer can have one or more neurons. Generally, the $\mathrm{i}^{\text {th }}$ neuron of the input layer connects with the $\mathrm{j}^{\text {th }}$ neuron of the hidden layer by some specified weight, and connects the $\mathrm{j}^{\text {th }}$ neuron of the hidden layer to the $\mathrm{k}^{\text {th }}$ neuron of output layer by some specified weight. The weighting function is used to simulate and recognize the response relationship between features of fused image and corresponding feature from the source images.

From the previous study, it is proved that ANN is a powerful and self-adaptive method of pattern recognition as compared to traditional linear and simple nonlinear analysis [20], [21]. Li et al. describes the application of ANN to pixel-level image fusion of multi-focus images taken from the same scene [22]. Sahoolizadeh et al. proposed a new hybrid approach for face recognition using Gabor wavelets and neural networks [23].

The first step of ANN-based image fusion is to decompose the registered images into several blocks with a specified size. Then, the features from the images of the corresponding blocks are extracted, and the normalized feature vector incident to neural network is constructed [24]. The features generally used are spatial frequency, visibility, edge, etc. After constructing and training the neural network, the ANN model can remember a functional relationship and can be used for further calculations. Hence, the ANN concept has been adopted to develop strongly nonlinear models for multisensor data fusion.

Rong et al. presented a feature-level image fusion method based on segmentation region and neural networks. The results indicate that this combined fusion scheme is better than the traditional methods [25]. The learning capability of neural networks makes ANN-based fusion methods feasible to customize the image fusion process as ANN-based fusion method exploits the pattern recognition capabilities of artificial neural networks. Many applications indicate that the ANN-based fusion methods have more advantages than traditional methods, especially when the input multiple sensor data were incomplete or with much noise. 
Signal \& Image Processing : An International Journal (SIPIJ) Vol.3, No.4, August 2012

\subsection{Features Selection}

In feature-level image fusion, the selection of different features is an important task. The five different features used to characterize the information level contained in a specific portion of the image are contrast visibility, spatial frequency, variance, energy of gradient (EOG), and edge information. R.Maruthi and Dr.K.Sankarasubramanian proposed a fusion procedure by using a selection mode according to the magnitude of the spatial frequency and visibility [26].

Contrast Visibility: It calculates the deviation of a block of pixels from that block's mean value. Hence, it relates to the clearness level of the block. The visibility of the image block is obtained using equation (1).

$$
\mathrm{VI}=\frac{1}{\mathrm{~m} * \mathrm{n}} \sum_{(\mathrm{i}, \mathrm{j}) \in \mathrm{B}_{\mathrm{k}}} \frac{\left|\mathrm{I}(\mathrm{i}, \mathrm{j})-\mu_{\mathrm{k}}\right|}{\mu_{\mathrm{k}}}
$$

Here $\mu \mathrm{k}$ and $\mathrm{m}^{*} \mathrm{n}$ are the mean and size of the block $\mathrm{B}_{\mathrm{k}}$ respectively.

Spatial Frequency: It measures the activity level in an image. It is used to calculate the frequency changes along rows and columns of the image. Spatial frequency is measured using equations (2), (3) and (4).

$$
\begin{aligned}
S F & =\sqrt{(R F)^{2}+(C F)^{2}} \\
\text { Where } R F & =\sqrt{\frac{1}{m * n} \sum_{i=1}^{m} \sum_{j=2}^{n}[I(i, j)-I(i, j-1)]^{2}} \\
C F & =\sqrt{\frac{1}{m * n} \sum_{j=1}^{n} \sum_{i=2}^{m}[I(i, j)-I(i-1, j)]^{2}}
\end{aligned}
$$

Here $\mathrm{I}$ is the image and $\mathrm{m}^{*} \mathrm{n}$ is the image size. A bigger value of spatial frequency describes the large information level in the image and therefore it measures the clearness of the image.

Variance: It measures the extent of focus in an image block. It is calculated using equation (5).

$$
\text { Variance }=\frac{1}{m * n} \sum_{i=1}^{m} \sum_{j=1}^{n}[I(i, j)-\mu]^{2}
$$

Here $\mu$ is the mean value of the block image and $m^{*} n$ is the image size. A high value of variance shows the greater extent of focus in the image block.

Energy of gradient (EOG): EOG is used to measure the amount of focus in an image. It is calculated using equation (6).

$$
\begin{aligned}
& E O G=\sum_{i=1}^{m-1} \sum_{j=1}^{n-1}\left(f_{i}^{2}+f_{j}^{2}\right) \\
& \text { where } f_{i}=f(i+1, j)-f(i, j) \\
& \text { and } f_{j}=f(i, j+1)-f(i, j)
\end{aligned}
$$

Here $\mathrm{m}$ and $\mathrm{n}$ represent the dimensions of the image block. A high value of energy of gradient shows greater amount of focus in the image block. 
Signal \& Image Processing : An International Journal (SIPIJ) Vol.3, No.4, August 2012

Edge information: The Canny edge detector is used to identify the edge pixels in the image block. It returns 1 if the current pixel belongs to some edge in the image otherwise it returns 0 . The edge feature is just the number of edge pixels contained within the image block.

\subsection{Proposed BFCN Method}

The proposed BFCN method decomposes both the source images into several blocks. Next, the discussed features in section 3.1 are extracted from the corresponding blocks and the normalized feature vector incident to $\mathrm{NN}$ is constructed. The $\mathrm{NN}$ is first trained by using few random index values and then simulated with feature vector index values. Finally, the inverse transform is applied.

\subsubsection{Stepwise working of the proposed BFCN method}

The block diagram of the proposed BFCN method is shown below in Figure 1.

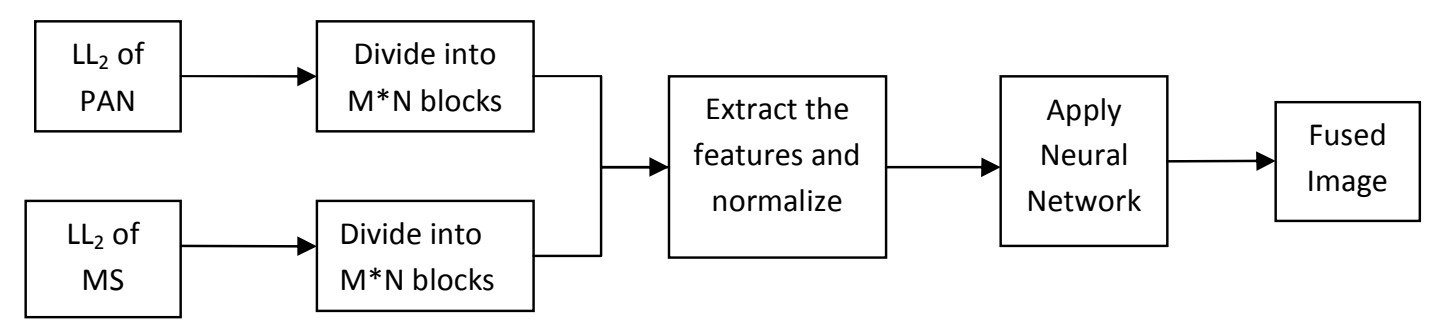

Figure 1. Block diagram of the proposed BFCN method

BFCN Algorithm:

begin

step 1: $\quad$ Read PAN and MS image.

step 2: $\quad$ Apply second level decomposition to both the source images.

step 3: Consider the low-low subcomponent of both the images. (For convenience, denote it as $\mathrm{LL}_{2}$ subcomponent).

step 4: $\quad$ Partition the $\mathrm{LL}_{2}$ subcomponent of both the images into $\mathrm{k}$ blocks of $\mathrm{M}^{*} \mathrm{~N}$ size and extract the features from every block. The features under study are contrast visibility, spatial frequency, energy of gradient, variance and edge information.

step 5: $\quad$ Subtract the feature values of $\mathrm{j}^{\text {th }}$ block of $\mathrm{LL}_{2}$ subband of PAN image from the corresponding feature values of $j^{\text {th }}$ block of $\mathrm{LL}_{2}$ subband of MS image. If the difference is 0 then denote it as 1 else -1 .

step 6: Construct an index vector for classification which will be given as an input for the neural network.

step 7: $\quad$ Create a neural network with three layers and adequate number of neurons. Train the newly constructed neural network with random index value.

step 8: $\quad$ Simulate the neural network with the feature vector index value. 
Signal \& Image Processing : An International Journal (SIPIJ) Vol.3, No.4, August 2012

step 9: $\quad$ If the simulated output $>1$ then the $\mathrm{j}^{\text {th }}$ subblock of $\mathrm{LL}_{2}$ subband of PAN image is considered else the $\mathrm{j}^{\text {th }}$ subblock of $\mathrm{LL}_{2}$ subband of MS image is considered.

Step 10: Reconstruct the entire block and apply inverse transform to get back the fused image.

end

\section{QUALITY ASSESSMENT TECHNIQUES}

To assess the quality of fused image, some quality measures are required. Goal of image quality assessment is to supply quality metrics that can predict perceived image quality automatically. While visual inspection has limitation due to human judgment, quantitative approach based on the evaluation of "distortion" in the resulting fused image is more desirable for mathematical modelling.

\subsection{Qualitative Measures}

The goals of the quantitative measures are normally used for the result of visual inspection due to the limitations of human eyes. In Mathematical modelling, quantitative measure is desirable. One can develop quantitative measure to predict perceived image quality. The quality assessment using noise-based measures are used to evaluate the noise of the fused image compared to the original MS image. The following optimal noise-based measures are implemented to judge the performance of fusion methods [27] as follows:

Peak signal to noise ratio (PSNR): PSNR is used to reveal the radiometric distortion of the fused image compared to the original image. It is calculated by using equation (7).

$$
\operatorname{PSNR}(d B)=10 \log _{10}\left(\frac{255 * 255}{M S E}\right)
$$

where MSE is the mean squared error and is used to measure the spectral distortion in the fused image. It is defined by using equation (8)

$$
\operatorname{MSE}=\frac{\sum_{\mathrm{i}=1}^{\mathrm{M}} \sum_{\mathrm{j}=1}^{\mathrm{N}}\left(\mathrm{I}_{\mathrm{R}}(\mathrm{i}, \mathrm{j})-\mathrm{I}_{\mathrm{F}}(\mathrm{i}, \mathrm{j})^{2}\right)}{\mathrm{M} * \mathrm{~N}}
$$

where $I_{R}(i, j)$ denotes pixel $(i, j)$ of the image reference and $I_{F}(i, j)$ denotes pixel $(i, j)$ of the fused image, $\mathrm{M} * \mathrm{~N}$ is the image size.

Mutual information measure (MIM): MIM is used to furnish the amount of information of one image in another. Given two images $\mathrm{M}(\mathrm{i}, \mathrm{j})$ and $\mathrm{N}(\mathrm{i}, \mathrm{j})$, it is defined using equation (9).

$$
\mathrm{I}_{\mathrm{MN}}=\sum_{x, y} P_{M N}(x, y) \log \frac{P_{M N}(x, y)}{P_{M}(x) P_{N}(y)}
$$

where $\mathrm{P}_{\mathrm{M}}(\mathrm{x})$ and $\mathrm{P}_{\mathrm{N}}(\mathrm{y})$ are the probability density functions of the individual images and $\mathrm{P}_{\mathrm{MN}}(\mathrm{x}, \mathrm{y})$ is joint probability density function.

Fusion Factor(FF) : FF is defined using equation (10).

$$
\mathrm{FF}=\mathrm{I}_{\mathrm{AF}}+\mathrm{I}_{\mathrm{BF}}
$$

where $\mathrm{A}$ and $\mathrm{B}$ are given two images and $\mathrm{F}$ is the fused image. A higher value of FF indicates that fused image contains moderately good amount of information present in both the images. 
Signal \& Image Processing : An International Journal (SIPIJ) Vol.3, No.4, August 2012

Standard Deviation (SD): SD is used to measure the contrast in the fused image and it is defined using equation (11). A well contrast image has high SD.

$$
S D=\sqrt{\sum_{i=0}^{L}\left(i-i^{\prime}\right)^{2} h_{F}(i)}
$$

where $i^{\prime}=\sum_{i=0}^{L} i h_{F}$ and $h_{F}$ is the normalized histogram of fused image and $\mathrm{L}$ is the number of gray levels.

Mean Absolute Error (MAE): MAE is used to measure the average magnitude of the errors in a set of forecasts, without considering their direction. It measures accuracy for continuous variables using equation (12).

$$
M A E=\frac{1}{M * N} \sum_{x=0}^{M-1} \sum_{y=0}^{N-1}\left(I_{R}(x, y)-I_{F}(x, y)\right)
$$

where $I_{R}(x, y)$ denotes pixel $(x, y)$ of the image reference and $I_{F}(x, y)$ denotes pixel $(x, y)$ of the fused image, $M * N$ is the image size.

\section{RESUlTS AND DISCUSSIONS}

In this paper, two kinds of fusion methods are implemented and executed on PC with $2.4 \mathrm{G} \mathrm{CPU}$ and 2.0 G RAM using Matlab 7.6.0 to compare their fusion results. The experiment is conducted and tested on IRS-1D PAN and LISS III images for the locations Hyderabad, Vishakhapatnam, Mahaboobnagar, and Patancheru in India and on Landsat 7 and QuickBird image data.

An efficient block based feature level wavelet transform with neural network (BFWN) method was proposed by us [28], which integrated wavelet transform with neural network. The present proposed BFCN method is compared with our previously proposed BFWN method for fusing the images about the locations Hyderabad, Vishakhapatnam, Mahaboobnagar, Patancheru, Landsat 7 and QuickBird images using the different quality assessment metrics discussed in 4.1.

The following Figure 2 represents the fused images for the location Hyderabad using the methods BFWN and the proposed BFCN.

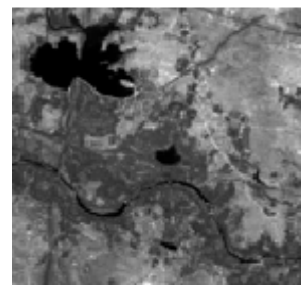

(a)

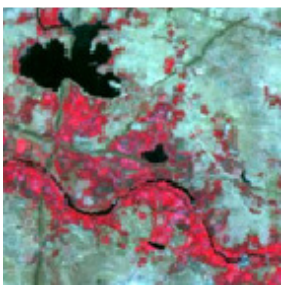

(b)

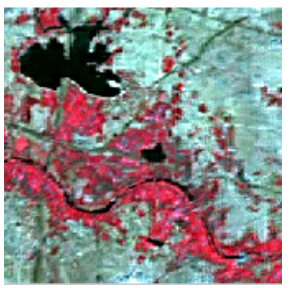

(c)

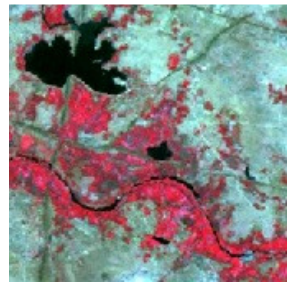

(d)

Figure 2. (a) PAN image (b) MS image (c) BFWN (d) BFCN

The following Figure 3 represents the fused images for the location Vishakapatnam using the methods BFWN and the proposed BFCN. 
Signal \& Image Processing : An International Journal (SIPIJ) Vol.3, No.4, August 2012

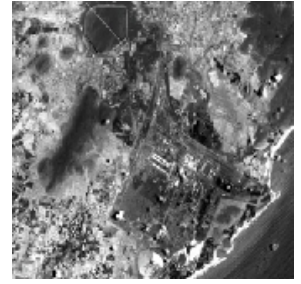

(a)

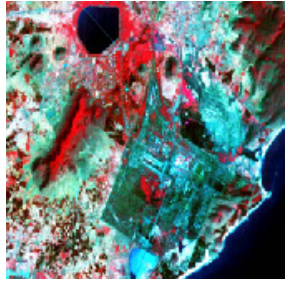

(b)

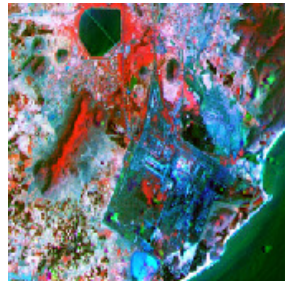

(c)

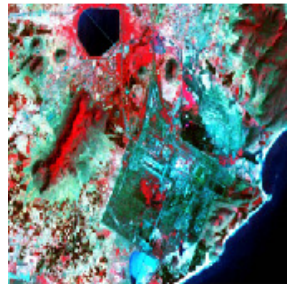

(d)

Figure 3. (a) PAN image (b) MS image (c) BFWN (d) BFCN

The following Figure 4 represents the fused images for the location Mahaboobnagar using the methods BFWN and the proposed BFCN.

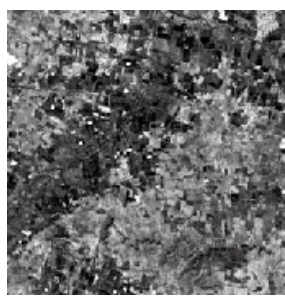

(a)

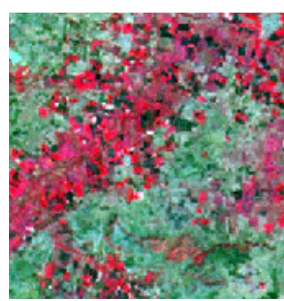

(b)

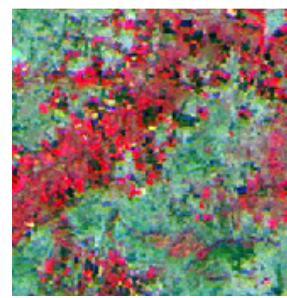

(c)

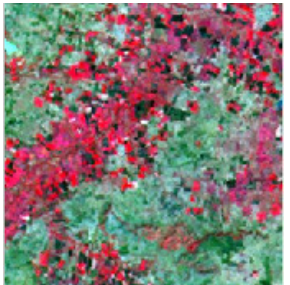

(d)

Figure 4. (a) PAN image (b) MS image (c) BFWN (d) BFCN

The following Figure 5 represents the fused images for the location Mahaboobnagar using the methods BFWN and the proposed BFCN.

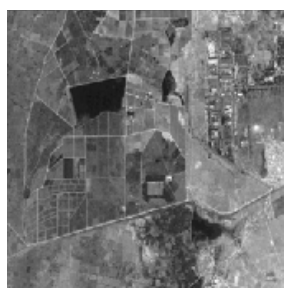

(a)

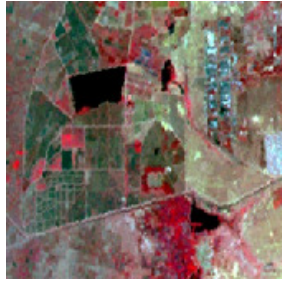

(b)

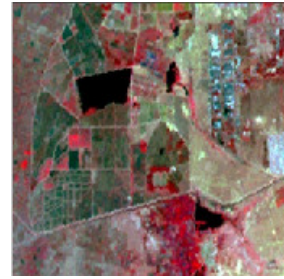

(c)

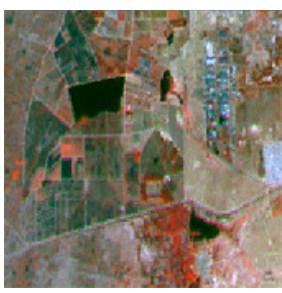

(d)

Figure 5. (a) PAN image (b) MS image (c) BFWN (d) BFCN

The following Figure 6 represents the fused images for the location Mahaboobnagar using the methods BFWN and the proposed BFCN.

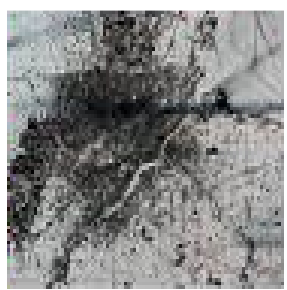

(a)

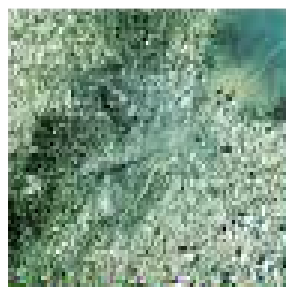

(b)

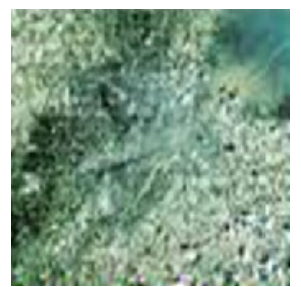

(c)

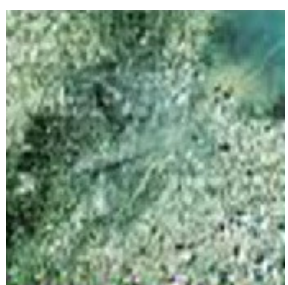

(d) 
Signal \& Image Processing : An International Journal (SIPIJ) Vol.3, No.4, August 2012
Figure 6
(a) PAN image
(b) MS image
(c) BFWN
(d) BFCN

The following Figure 7 represents the fused images for the location Mahaboobnagar using the methods BFWN and the proposed BFCN.

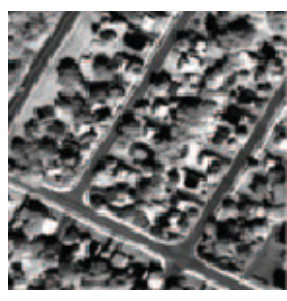

(a)

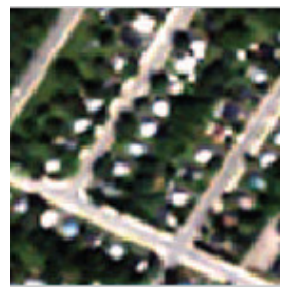

(b)

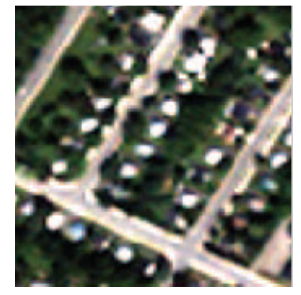

(c)

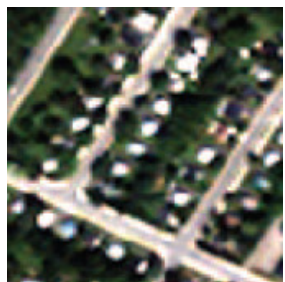

(d)

Figure 7. (a) PAN image (b) MS image (c) BFWN (d) BFCN

The calculated values of PSNR, MIM, FS, SD and MAE for the methods BFWN and the proposed BFCN about the locations Hyderabad, Vishakhapatnam and Mahaboobnagar image data sets are mentioned in Table 1 and for the locations Patancheru, Landsat 7 and QuickBird image data sets are mentioned in Table 2 .

Table 1: Comparison of different metrics using BFWN and BFCN methods for Hyderabad image, Vishakhapatnam image and Mahaboobnagar image.

\begin{tabular}{|c|c|c|c|c|c|c|}
\hline \multirow{2}{*}{$\begin{array}{l}\text { Quality } \\
\text { metrics }\end{array}$} & \multicolumn{2}{|c|}{ Hyderabad Image } & \multicolumn{2}{l|}{ Vishakhapatnam Image } & \multicolumn{2}{l|}{ Mahaboobnagar Image } \\
\cline { 2 - 7 } & BFWN & BFCN & BFWN & BFMN & BFWN & BFCN \\
\hline PSNR & 77.0658 & $\mathbf{8 1 . 8 3 7 0}$ & 70.5083 & $\mathbf{7 4 . 6 2 7 8}$ & 72.3011 & $\mathbf{7 4 . 3 0 1 1}$ \\
\hline MIM & 2.0289 & $\mathbf{2 . 6 2 8 9}$ & 1.0235 & $\mathbf{2 . 1 6 8 7}$ & 1.0060 & $\mathbf{1 . 6 4 2 6}$ \\
\hline FF & 4.0577 & $\mathbf{4 . 0 5 9 7}$ & 2.0470 & $\mathbf{4 . 3 3 7 3}$ & 2.0120 & $\mathbf{3 . 2 8 5 1}$ \\
\hline SD & 40.5197 & $\mathbf{4 0 . 5 1 9 0}$ & 62.3872 & $\mathbf{6 2 . 3 8 7 0}$ & 49.4440 & $\mathbf{4 9 . 4 4 3 6}$ \\
\hline MAE & 0.0161 & $\mathbf{0 . 0 0 6 1}$ & 0.0357 & $\mathbf{0 . 0 2 0 9}$ & 0.0291 & $\mathbf{0 . 0 2 1 1}$ \\
\hline
\end{tabular}

Table 2 : Comparison of different metrics using BFWN and BFCN methods for Patancheru image, Landsat 7 image and QuickBird image.

\begin{tabular}{|c|c|c|c|c|c|c|}
\hline \multirow{2}{*}{$\begin{array}{l}\text { Quality } \\
\text { metrics }\end{array}$} & \multicolumn{2}{|l|}{ Patancheru Image } & \multicolumn{2}{l|}{ Landsat 7 Image } & \multicolumn{2}{c|}{ QuickBird Image } \\
\cline { 2 - 7 } & BFWN & BFCN & BFWN & BFCN & BFWN & BFCN \\
\hline PSNR & 73.8269 & $\mathbf{7 6 . 6 0 1 0}$ & 73.4789 & $\mathbf{7 8 . 2 5 0 1}$ & 75.4508 & $\mathbf{7 5 . 9 5 0 8}$ \\
\hline MIM & 1.1985 & $\mathbf{1 . 6 4 3 7}$ & 1.6842 & $\mathbf{1 . 6 8 4 5}$ & 1.8487 & $\mathbf{1 . 9 4 8 7}$ \\
\hline
\end{tabular}


Signal \& Image Processing : An International Journal (SIPIJ) Vol.3, No.4, August 2012

\begin{tabular}{|c|c|c|c|c|c|c|}
\hline FF & 2.3970 & $\mathbf{3 . 2 8 7 3}$ & 3.3684 & $\mathbf{3 . 3 6 8 6}$ & 3.6974 & $\mathbf{3 . 6 9 9 4}$ \\
\hline SD & 35.4804 & $\mathbf{3 5 . 4 8 0 0}$ & 39.6275 & $\mathbf{3 9 . 6 2 7 0}$ & 77.5713 & 77.5713 \\
\hline MAE & 0.0258 & $\mathbf{0 . 0 1 0 1}$ & 0.0296 & $\mathbf{0 . 0 2 9 4}$ & 0.0194 & $\mathbf{0 . 0 1 7 4}$ \\
\hline
\end{tabular}

By comparing the values of PSNR for fusing the six pairs of data images using the methods BFWN and BFCN, the results show that higher value of PSNR is achieved for the proposed BFCN method. This graph is depicted in the following Figure 8. Similarly, by comparing the values of SD for fusing the six pairs of data images, the results show that smaller value of SD is achieved for the proposed BFCN method for five image data sets i.e., Hyderabad, Vishakhapatnam, Mahaboobnagar, Patancheru and Landsat 7. The SD value for both the methods is same for QuickBird data images. This graph is depicted in the following Figure 9.

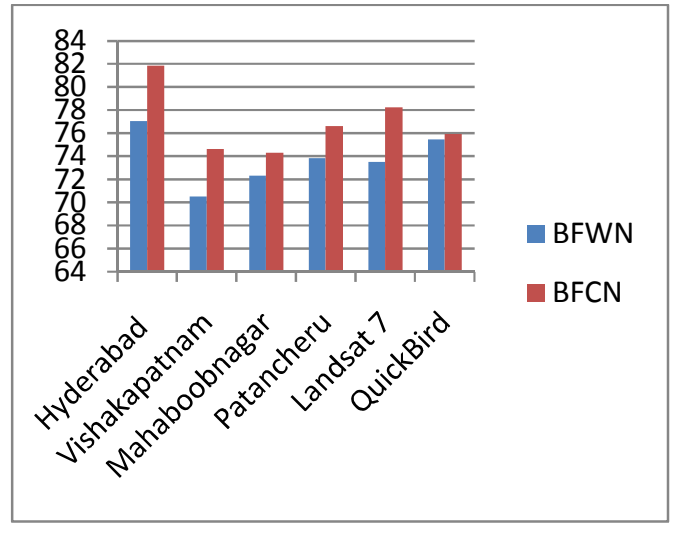

Figure 8. Comparing PSNR values

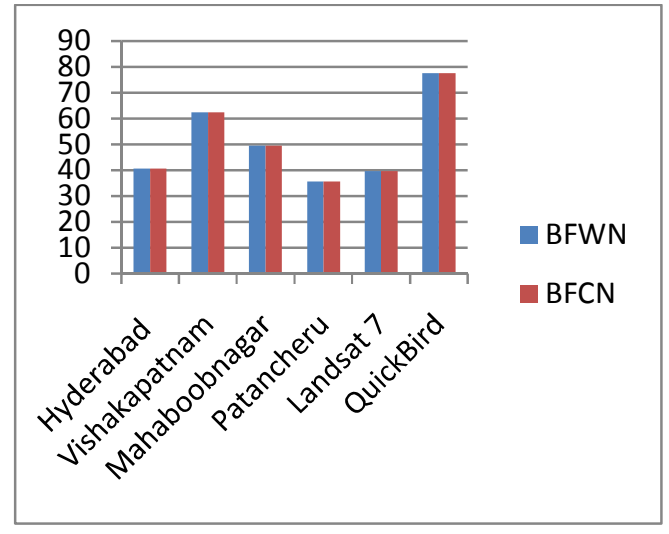

Figure 9. Comparing SD values

\section{CONCLUSIONS}

The potentials of image fusion using the proposed BFCN method are explored. The various fusion results are analyzed by using quality performance metrics. The higher value for PSNR, MIM and FF is achieved for the proposed BFCN method for all the six image data sets. The higher value of PSNR implies that, the spectral information in MS image is preserved effectively and high signal is also preserved. The higher value of MIM and FF indicate that symmetry is achieved by retaining the spectral information. The higher value of FF indicates that fused image contains moderately good amount of information present in both the images. Among six pairs of images, the smaller value of SD is obtained for the proposed BFCN method for the five image data sets i.e., Hyderabad, Vishakhapatnam, Mahaboobnagar, Patancheru and Landsat 7. The smaller value of SD indicates that not much deviation is induced in the fused image. For all the six image data sets, the smaller value for MAE is obtained for the proposed BFCN method. The smaller value of MAE indicates that error rate is reduced in the fused image. The metric parameters PSNR, MIM and FF are maximum for the proposed BFCN method, and SD and MAE are minimum for the proposed BFCN method. The experimental results indicate that BFCN outperforms our earlier BFWN method. Hence, it is ascertained that contourlet transform with $\mathrm{NN}$ method has superior performance than wavelet transform with NN method. The results are verified for LISS III images and the study can be extended for other types of images. 
Signal \& Image Processing : An International Journal (SIPIJ) Vol.3, No.4, August 2012

\section{REFERENCES}

[1] V Petrović, C Xydeas, "Computationally Efficient Pixel-level Image Fusion”, Proc. Eurofusion99, October 1999., pp 177-184.

[2] H Li, S Munjanath, S Mitra, "Multisensor Image Fusion Using the Wavelet Transform”, Graphical Models and Image Proc., Vol. 57, No. 3, 1995, pp 235-245.

[3] Abdul Basit Siddiqui, M.Arfan Jaffar, Ayyaz Hussain, Anwar M. Mirza, "Block-based Featurelevel Multi-focus Image Fusion”, 5th International Conference on Future Information Technology (FutureTech), 2010 IEEE, 978-1-4244-6949-9/10.

[4] G. Piella, "A region-based multiresolution image fusion algorithm”, Information Fusion, 2002. Proceedings of the Fifth International Conference on Information Fusion, 1557 - 1564 vol.2

[5] A. Toet, "Image fusion by a ratio of low pass pyramid" in Pattern Recognition Letters, vol. 9, no. 4, pp. 245-253, 1989.

[6] K. Kannan, S. Arumuga Perumal, K. Arulmozhi, "Performance Comparison of various levels of Fusion of Multi-focused Images using Wavelet Transform", 2010 International Journal of Computer Applications (0975 - 8887), Volume 1 - No. 6.

[7] Jiang Dong, Dafang Zhuang, Yaohuan Huang and Jingying Fu, "Advances in Multi-sensor data fusion : algorithm and applications", Sensors 2009, 9, 7771-7784; doi:10.3390/s91007771.

[8] Negar Riazifar, and Mehran Yazdi, "Effectiveness of Contourlet vs Wavelet Transform on Medical Image Compression: a Comparative Study", World Academy of Science, Engineering and Technology, 49, 2009.

[9] Ramin Eslami and Hayder Radha, "New Image Transforms Using Hybrid Wavelets and Directional Filter Banks: Analysis and Design".

[10] Ramin Eslami and Hayder Radha, "Wavelet-based Contourlet Coding Using an SPIHT - like Algorithm”, IEEE International Conference on Image Processing, Oct, 2004.

[11] Maziyar Khosravi, Mazaheri Amin, "Block feature based image fusion using multiwavelet transforms" - International Journal of Engineering Science and Technology (IJEST) - ISSN : 0975-5462, vol. 3, no. 8, August 2011, 6644.

[12] Jean-Luc Starck, Emmanuel J. Candès, and David L. Donoho, “The Curvelet Transform for Image Denoising”, IEEE TRANSACTIONS ON IMAGE PROCESSING, vol. 11, no. 6, June 2002.

[13] V. Velisavljevic, P. L. Dragotti, M. Vetterli, "Directional Wavelet Transforms and Frames", Proceedings of IEEE International Conference on Image Processing (ICIP2002), vol. 3, pp. 589592, Rochester, USA, September 2002.

[14] M. N. Do and M. Vetterli, "The contourlet transform: An efficient directional multiresolution image representation," IEEE Transactions on Image Processing, vol. 14, no. 12, pp. 2091-2096, Dec 2005.

[15] N. Kingsbury, "Complex wavelets for shift invariant analysis and filtering of signals", Journal of Applied and Computational Harmonic Analysis, 10 (2001), pp. 234-253.

[16] E.J. Candés, D.L. Donoho, "Curvelets - a surprisingly effective nonadaptive representation for objects with edges", A. Cohen, C. Rabut, L.L. Schumaker (Eds.), Curve and Surface Fitting, Vanderbilt University Press, Saint-Malo (2000).

[17] Minh N. Do, M. Vetterli, "Contourlet: a new directional multiresolution image representation", IEEE Transactions on Image Processing, 1 (2002), pp. 1497-1501.

[18] M. N. Do, M. Vetterli, "The contourlet transform: An efficient directional multiresolution image representation”, IEEE Transactions on Image Processing, no. 12, pp. 2091-2106, 2005.

[19] Ding Li and Han Chongzhao, "Multi-focus image fusion using wavelet based contourlet transform and region”, 2009 International Conference on Information Management and Engineering. 
Signal \& Image Processing : An International Journal (SIPIJ) Vol.3, No.4, August 2012

[20] Louis, E.K.; Yan, X.H. “A neural network model for estimating sea surface chlorophyll and sediments from thematic mapper imagery”. Remote Sens. Environ. 1998, 66, 153-165.

[21] Dong. J.; Yang, X.; Clinton, N.; Wang, N. “An artificial neural network model for estimating crop yields using remotely sensed information”. Int. J. Remote Sens. 2004, 25, 1723-1732.

[22] Shutao, L.; Kwok, J.T.; Yaonan W. "Multifocus image fusion using artificial neural networks". Pattern Recognit. Lett. 2002, 23, 985-997.

[23] Hossein Sahoolizadeh, Davood Sarikhanimoghadam, and Hamid Dehghani, "Face Detection using Gabor Wavelets and Neural Networks", World Academy of Science, Engineering and Technology 45, 2008.

[24] Shutao, L.; Kwok, J.T.; Yaonan W. "Multifocus image fusion using artificial neural networks". Pattern Recognit. Lett. 2002, 23, 985-997.

[25] Wang, R.; Bu, F.L.; Jin, H.; Li, L.H. "A feature-level image fusion algorithm based on neural networks". Bioinf. Biomed. Eng. 2007, 7, 821-824.

[26] R.Maruthi, and Dr.K.Sankarasubramanian, "Multi focus image fusion based on the Information level in the regions of the images", Journal of Theoretical and Applied Information Technology, (C) 2005 - 2007 JATIT.

[27] H.Li, S. Manjunath and S.K. Mitra, "Multi-sensor image fusion using the wavelet transform" in Graphical Models and Image Processing, vol. 57, no.3, pp. 235-245, 1995.

[28] C.M.Sheela Rani, V.VijayaKumar, B.Sujatha "An Efficient Block based Feature level Image fusion Technique using Wavelet transform and Neural network", International Journal of Computer Applications, 0975-8887, August 2012. 